\title{
Comparação de técnicas coproparasitológicas para a contagem de ovos de nematóides gastrintestinais de ovinos
}

\section{Comparison between coproparasitologic techniques for egg counting of gastrointestinal nematodes of sheep}

\author{
Marcelo Abidu, ${ }^{\star}$ Richard Karl Reinecke, ${ }^{* *}$ Dalton Garcia de Mattos Júnior, ${ }^{* * *}$ Paula Amorim Schiavo ${ }^{* * * *}$
}

\begin{abstract}
Resumo
Foi realizado um estudo comparativo entre três técnicas copro-parasitológicas para a contagem de ovos dos principais gêneros de nematóides gastrintestinais de ovinos. Coletaram-se fezes de 50 ovinos adultos de ambos os sexos, naturalmente infectados. Cada amostra de fezes foi processada cinco vezes para cada técnica proposta. Através da análise estatística, foi demonstrado que uma nova técnica (Reinecke et al., 1992) foi a mais sensível na detecção de ovos de nematoídes gastrintestinais, quando comparada com as técnicas de Figueiredo et al. (1984) e de Whitlock (1948).
\end{abstract}

Palavras-chave: ovinos; nematóides; comparação de técnicas.

\begin{abstract}
A field comparative study were realized comparing three coproparasitologic techniques for egg counting of gastrointestinal nematodes of sheep. Fifty sheep faeces samples were analysed five times by each proposed technique. After being processed, the statistical analysis of the results showed that the Visser Filter was the most sensitive technique for egg counting of gastrointestinal nematodes of sheep.
\end{abstract}

Keywords: sheep; nematode; techniques comparison.

\section{Introdução}

A infecção helmíntica nos animais domésticos apresenta-se como um dos grandes obstáculos para o melhoramento da produção animal, tão necessária para prover o aumento de proteína animal.

O conhecimento exato das perdas econômicas ocasionadas pelos helmintos não é fácil de quantificar, já que geralmente as infecções são crônicas, resultando em uma alta morbidade e baixa mortalidade dos animais parasitados (McAuliffe, 1977).

Já as infecções maciças por alguns gêneros de helmintos causam enfermidade aguda com alta mortalidade e as perdas econômicas podem ser melhor calculadas, principalmente nos animais jovens.

As conseqüências das infecções por helmintos gastrintestinais sobre os animais são as mais variadas, podendo ser resumidas nas seguintes: danos à mucosa do abomaso e intestinos, hematofagismo, competição com o hospedeiro por minerais e outros nutrientes, diminuição do apetite e perda de peso. Esses efeitos se fazem notar principalmente pelo baixo índice de desenvolvimento dos animais, retardando o abate em um ou dois anos e pelo aumento da taxa de mortalidade do rebanho (McAuliffe, 1977).

Tão importante quanto conhecer os efeitos e prejuízos provocados pelas helmintoses é saber o grau em que a mesma está ocorrendo, e por isso uma das maneiras é aplicar as técnicas que quantificam ovos por grama de fezes (opg), que é uma prática laboratorial muito utilizada, não só para verificar a sanidade do rebanho, correlacionando o opg com a carga parasitária, bem como verificar a eficiência de produtos anti-helmínticos mediante a redução do opg (Ueno, Gutierres, 1983).

Visando o aperfeiçoamento do diagnóstico das helmintoses gastrintestinais dos animais domésticos, alguns pesquisadores têm sido motivados a criar ou mesmo modificar técnicas já existentes, como é o caso de Whitlock (1948), Figueiredo et al. (1984) e Reinecke et al. (1992). Assim, estas três técnicas são

\footnotetext{
* Med. Vet. Prof. Assist. DBA-IB-UFRRJ BR 465, Km 7, s/n, Seropédica, RJ - CEP 23851-970. E-mail: abidu_m@yahoo.com.

${ }^{\star *}$ Med. Vet. Prof. Emérito da Univ. de Pretória, África do Sul.

*** Med. Vet. Prof.Adjunto do Dep. de Patologia e Clínica. Faculdade de Veterinária da UFF.

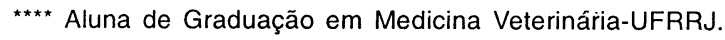


comparadas no presente trabalho com o objetivo de avaliar a eficácia da técnica de Reinecke et al. (1992) no diagnóstico das helmintoses gastrintestinais de ovinos.

\section{Material e métodos}

O experimento foi realizado nas dependências do Laboratório de Parasitologia - Convênio UFRRJ/EMBRAPA - SANIDADE ANIMAL, no município de Seropédica-RJ.

Com o auxílio de um tubo plástico medindo $23 \mathrm{~cm}$ de comprimento e $3,5 \mathrm{~cm}$ de diâmetro interno, cortado em forma de bisel em sua extremidade distal (Figura 1), foram coletadas, individualmente, diretamente da ampola retal, cíbalas de 50 ovinos adultos, de ambos os sexos, naturalmente infectados por nematóides gastrintestinais.

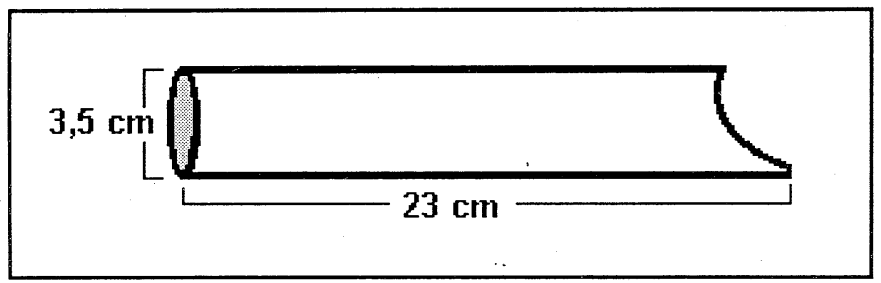

Figura 1: Tubo plástico para coleta de cíbalas

As cíbalas foram, então, vertidas para sacos plásticos individuais, etiquetadas e acondicionadas em caixas isotérmicas contendo gelo, e remetidas imediatamente ao laboratório.

No laboratório, cada amostra de fezes foi homogeneizada, pesada e separada para a realização das técnicas de Whitlock (1948), Figueiredo et al. (1984) e Reinecke et al. (1992).

Estas técnicas foram repetidas cinco vezes em cada um dos animais examinados, e os resultados do opg comparados.

\section{Análise estatística}

A análise estatística (Tabela 1 ) dos resultados foi realizada através do computador com assessoramento técnico do Centro de Processamento de Dados da Universidade Federal Fluminense. Foram utilizados procedimentos extraídos do software SAS (Statistical Analysis System), aplicando-se o teste de Wilcoxon, segundo Campos (1983).

Tabela 1: Análise estatística dos resultados segundo o teste de Wilcoxon

\begin{tabular}{lcc}
\hline Técnica & Soma dos escores & Escore médio \\
\hline WHITLOCK & 3981,50 & 79,63 \\
REINECKE & 4871,00 & 97,42 \\
FIGUEIREDO & 2472,50 & 49,45 \\
\hline
\end{tabular}

\section{Resultados e discussão}

$\mathrm{Na}$ tabela 2 estão os resultados das três técnicas.
Tabela 2: Resultado da comparação das três técnicas

\begin{tabular}{|c|c|c|c|}
\hline Techica & Whitlock & Feinecke & Figueiredo \\
\hline$N^{0}$ de amostras & 50 & 50 & 50 \\
\hline$N^{\circ}$ de repetições & 5 & 5 & 5 \\
\hline Percentual de Amostras positivas & 84 & 96 & 94 \\
\hline OPG médio & 1713,5 & 1958,14 & 411,24 \\
\hline
\end{tabular}

A técnica de Reinecke et al.(1992) demonstrou ser a mais sensivel para detectar ovos de nematóides gastrintestinais de ovinos, uma vez que apresentou um escore médio superior, revelando que, dos 50 animais examinados, apenas dois apresentavam-se com opg negativo.

A técnica de Whitlock (1948) demonstrou ser a técnica de pior sensibilidade, apesar de apresentar um número maior de escore médio quando comparado com a técnica de Figueiredo et al. (1984). Isto se deve ao fato de que, segundo a literatura, as técnicas de centrífugo-flutuação são mais sensíveis para opgs baixos, detectando níveis de parasitismo subclínicos (Stoll, 1923 ; Pino et al. 1981 ; Huber, Kerboeuf, 1984).

Resultados semelhantes foram observados por Rossanigo, Gruner (1991) quando compararam e recuperaram $93 \%$ de ovos pela técnica de centrífugo-flutuação contra $16,5 \%$ de ovos pela técnica de Whitlock.

Apesar da técnica de Whitlock apresentar valores de opgs superiores aos resultados obtidos por Figueiredo et al.(1984) refletindo na soma dos escores, esta técnica apresentou maior freqüência de resultados negativos nas amostras de fezes examinadas.

Entretanto, esses resultados confrontam com os de Levine et al. (1960), que obtiveram melhores resultados empregando a técnica de Whitlock quando comparada com a técnica de centrífugo-flutuação.

A técnica de Reinecke et al. (1992), utilizada na recuperação de ovos de nematóides de bovinos, adaptada no presente trabalho para ovinos, demonstrou ser a mais sensível na detecção de ovos nas fezes, quando comparadas às outras técnicas testadas.

Na técnica de Reinecke, os ovos dos helmintos passam por várias lavagens, o que facilita a identificação dos mesmos, assegurando um diagnóstico mais preciso. Essa técnica permite detectar, ao mesmo tempo, ovos de nematóides e trematódeos (Abidu et al., 1995).

Mais estudos devem ser realizados, comparando e avaliando a sensibilidade da presente técnica demonstrada, pois, através dela, poderão ser desenvolvidos vários estudos sobre a carga parasitária, eficiência de produtos anti-helmínticos e avaliação da resistência de diversos nematoídes a drogas comumente utilizadas, como citado por Reinecke (com. pessoal).

\section{Conclusão}

A técnica de Reinecke et al. (1992) modificada para ovinos é a técnica mais sensível para a detecção de ovos de nematóides gastrintestinais de ovinos quando comparada com as técnicas de Figueiredo et al. (1984) e Whitlock (1948), constituindo mais uma técnica para ser utilizada com segurança na rotina laboratorial. 


\section{Referências bibliográficas}

ABIDU, M., SCHERER, P.O., SERRA FREIRE, N.M. Diagnóstico Coprológico Quantitativo para Fasciola hepatica através do Filtro de Visser. Revista da Universidade Rural, Série Ciência da Vida. v. 17, n. 2, p. 103-105, 1995.

CAMPOS, H. Estatística experimental não-paramétrica. 4. ed. São PauIo: USP, 1983.

FIGUEIREDO, P.C., SERRA-FREIRE, N.M., GRISI, L. Eimerias de bovinos leiteiros no Estado do Rio de Janeiro: tecnica de diagnóstico e espécies identificadas. Atas Soc. Biol. Rio de Janeiro.v. 24, p. 22-26, 1984.

HUBER, J., KERBOEUF, D. A new method for culture of larvae used in diagnosis of ruminant gastrointestinal and strongylosis: Comparison with fecal cultures. Can J. Comp Med. v. 48, p. 63-71, 1984.

LEVINE, N.D., MEHRA, K.N., CLARK, D. T., AVES, I.J. A comparison of nematode egg counting technique for cattle and sheep faeces. Am. J. Vet Res., v. 21., p. 511-515, 1960.

McAULIFFE. P.R. The importance of worms to sheep and cattle. Worm Control Seminar-Hamilton Pastoral Research Station, october 1-3, 1977.
PINO, L.A., MORALES, G., RODRIGUES, E. Comparative study of the Stoll and Mc Master coproscopic techniques. Bol. Dir . Malariol. San. Amb, v. 21, n. 3/4, p. 192-195, 1981.

REINECKE, R.K., FIGUEIREDO, M. A., MATTOS, Jr., D.G. Um método simples para o diagnóstico dos nematóides gastrointestinais mais comuns de bezerros. CONGRESSO BRASILEIRO DE MEDICINA VETERINÁRIA, 22., Curitiba. 10-13 de novembro, 1992.

ROSSANIGO, C. E., GRUNER, L. Accurracy of two methods for couting eggs of sheep nematode parasites. Vet.Parasitol., v. 39, p. 115121, 1991.

STOLL, N.R. (1923) Investigations on the control of hookworm disease. $X V$. An effective method counting hookworm eggs in faeces. Am. J. Hyg. v. 3, n. 10, p. 59-70.

UENO, H., GUTIERRES, V. C. Manual para diagnóstico das helmintoses de ruminantes. JICA. 165 p. 1983.

WHITLOCK, H. V. Some modifications of the Mc Master helminth egg counting technique and apparatus. J. Counc. Sci. Ind. Res. v. 21, p. 177-180, 1948. 